\title{
Production and validation of primary carbon dioxide reference standards at INRIM
}

\author{
Francesca Rolle $^{1, *}$, Francesca Pennecchi $^{1}$, Enrica Pessana ${ }^{1}$ and Michela Sega ${ }^{1}$ \\ ${ }^{1}$ Istituto Nazionale di Ricerca Metrologica (INRIM), Strada delle Cacce 91, 10135, Torino, Italy
}

\begin{abstract}
The accurate and reliable determination of carbon dioxide $\left(\mathrm{CO}_{2}\right)$ levels in atmosphere is important for the role of this gas in the greenhouse effect. In addition, the rising level of $\mathrm{CO}_{2}$ absorbed by the oceans from the atmosphere causes an acidification of the marine waters, with fallouts on the global ecosystem. In this framework, the application of the metrological concepts is necessary to assure the reliability of the measurement results. At INRIM, activities for the preparation and verification of $\mathrm{CO}_{2}$ primary reference mixtures are carried out. A facility for the production of primary $\mathrm{CO}_{2}$ standards by gravimetry has been used since 2004. Due to the huge amount of gas mixtures that are needed to cover different ranges of molar fractions during the calibration of instrumentation, a new system for dynamic dilution was recently set up. An overview of the above-mentioned activities, with a focus on some crucial issues related to mixture verification and stability studies, is presented in this work.
\end{abstract}

\section{Introduction}

The accurate and reliable determination of carbon dioxide $\left(\mathrm{CO}_{2}\right)$ levels in atmosphere is important for the role of this gas in the greenhouse effect: the monitoring of $\mathrm{CO}_{2}$ trends is fundamental to obtain significant information about the current pollution levels and the future trends on a global scale. The choice of addressing the metrological traceability of $\mathrm{CO}_{2}$ measurement results derives from $\mathrm{CO}_{2}$ relevance for the World Meteorological Organisation (WMO), an intergovernmental organisation that promotes observation of atmospheric environment through programmes such as the "Global Atmosphere Watch" (GAW) [1].

At the same time, the rising level of atmospheric $\mathrm{CO}_{2}$ results in a higher amount of $\mathrm{CO}_{2}$ absorbed by the oceans, causing an acidification of the marine waters, with fallouts on the global ecosystem [2]. The partial pressure of $\mathrm{CO}_{2}\left(p \mathrm{CO}_{2}\right)$ in seawater is considered an Essential Climate Variable which can be monitored in situ with networked sensors. In this framework it is fundamental the establishment of metrologically sound approaches to assure the reliability of $p \mathrm{CO}_{2}$ measurement results.

The availability of suitable reference standards and proper analytical procedures represents the basis for a reliable quantification of $\mathrm{CO}_{2}$ in both the environmental compartments. This work presents the activities carried out at INRIM in this framework focusing on some crucial issues related to mixture verification and stability studies.

\section{Experimental activity}

At INRIM, $\mathrm{CO}_{2}$ primary reference mixtures are prepared and verified. A facility for the production of primary $\mathrm{CO}_{2}$ standards by gravimetry has been used since 2004 [3]. Primary reference mixtures of $\mathrm{CO}_{2}$ in matrices of nitrogen $\left(\mathrm{N}_{2}\right)$ or synthetic air are prepared by gravimetry in high pressure cylinders of aluminium alloy, having an internal volume of $5 \mathrm{~L}$. Figure 1 shows two primary reference gas mixtures produced at INRIM.

A system for dynamic dilution was recently set up [4] to cover different ranges of molar fractions both for instrument calibration and gravimetric mixture verifications.

The $\mathrm{CO}_{2}$ mixtures are prepared in a wide range of molar fractions, from $50 \mu \mathrm{mol} \mathrm{mol}^{-1}$ up to $15 \mathrm{cmol} \mathrm{mol}^{-1}$. Particular interest is paid to the preparation of $\mathrm{CO}_{2}$ reference gas mixtures at ambient level, thus covering the molar fraction range $(50-1000) \mu \mathrm{mol} \mathrm{mol}{ }^{-1}$. The relative standard uncertainty associated with these mixtures decreases with increasing molar fraction and is in the range $(0.5-0.01) \%$, for both the primary methods.

\footnotetext{
Corresponding author: f.rolle@inrim.it
}

C The Authors, published by EDP Sciences. This is an open access article distributed under the terms of the Creative Commons Attribution License 4.0 (http://creativecommons.org/licenses/by/4.0/). 


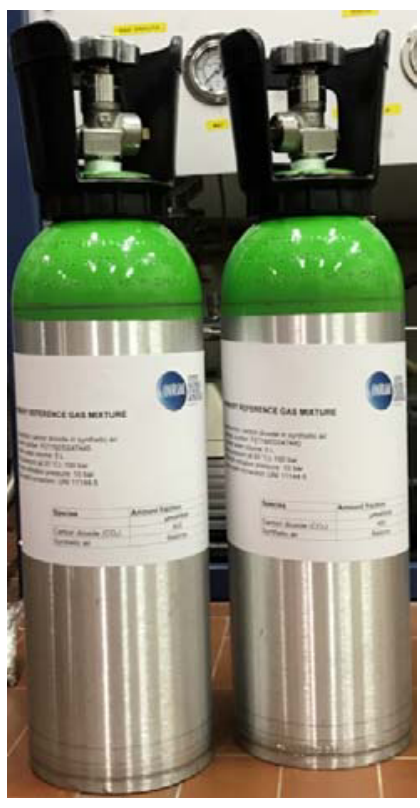

Fig. 1. Primary reference gas mixtures of $\mathrm{CO}_{2}$ in synthetic air gravimetrically prepared at INRIM.

\subsection{Primary methods for the preparation of reference gas mixtures}

\subsubsection{Gravimetry}

Gravimetry is a primary method which is used for the preparation of primary reference standards in high pressure cylinders by weighing and it gives direct traceability to mass standards and purity of the parent gases. The preparation procedure followed at INRIM is based on [5]. It consists of two parts: the conditioning and the preparation phase. During the second part of the procedure, the mass of the cylinder in which the mixture is being prepared (target cylinder) is compared with the mass of an empty reference cylinder carrying out a high precision weighing. Calibrated mass standards are arranged on the top of the lighter cylinder to maintain the difference between the target cylinder and the reference gas cylinder within $1 \mathrm{~g}$, thus optimising the mass comparator performance [6]. The use of calibrated mass standards also assures the metrological traceability of the mass measurement. The reference cylinder and the target cylinder are alternatively weighed according to the double substitution scheme (A-B-B-A) to minimise the correction for the buoyancy effect.

Each mixture is individually prepared: its gravimetric molar fraction and the associated uncertainty are assigned according to [5]. After the preparation, each mixture is analytically verified by means of a specific analyser calibrated with independent reference gas mixtures. The verification aims at validating the gravimetric procedure, also excluding the presence of gross mistakes during the preparation. The subsequent analyses of the mixtures are also used to determine the stability of the mixtures over time.

For these reasons, a large number of independent gas mixtures covering a wide range of molar fractions is needed. In addition, particular care has to be paid to the composition of the reference mixtures used to calibrate the analysers, both in terms of molar fraction of the analytes and of the matrix constituents. The whole preparation process is quite complex and time consuming. For these reasons, the set-up of an alternative and complementary procedure for a rapid and accurate preparation of metrologically traceable reference gas mixtures was implemented at INRIM, namely the dynamic dilution (see par. 2.1.2).

\subsubsection{Dynamic dilution}

Dynamic dilution is used to prepare in real time a gas mixture, by mixing two gases together in order to reach the desired molar fraction, starting from a more concentrated gas mixture and a matrix gas. With respect to gravimetry, dynamic dilution has the advantage that a gas mixture can be diluted in real time on a range of different molar fractions. The reference gases obtained by dynamic dilution can be used for instrumental calibration, hence are also useful to check and verify gas standards prepared by gravimetry.

At INRIM a dynamic dilution system composed of Mass Flow Controllers (MFCs) and a mixing chamber was developed. Different pairs of MFCs are used depending on the dilution needed to prepare mixtures at the desired molar fractions. MFCs are calibrated at INRIM obtaining polynomial regression curves which give a correction for the nominal flow displayed by the MFCs.

\subsection{Analytical verification and stability study}

Different analytical techniques can be used for the determination of the molar fractions of $\mathrm{CO}_{2}$. Non Dispersive Infrared (NDIR) photometry is an analytical technique widely used for $\mathrm{CO}_{2}$ determination, being a robust and selective one, easily applicable to portable instruments. A NDIR photometer Advance Optima Uras 14 (ABB, Switzerland) is used at INRIM for the verification of the molar fraction of the prepared mixtures. A three-point calibration is carried out choosing reference gas mixtures in a molar fraction range that covers the nominal analytical value. The calibration mixtures must be independent from the mixtures to be verified. Recently a NDIR calibration procedure involving the use of reference mixtures generated by dynamic dilution has been put in place. For the calibration curve determination, an approach based on Weighted Total Least Squares (WTLS) regression has been adopted. The WTLS has the advantage of taking into account uncertainties both on the independent and dependent variables. In particular, the software used, namely CCC-software [7], allows to directly obtain the analysis curve and the uncertainties associated with the regression parameters.

At present, research is ongoing to study the different interferences and undesired effects which can occur during the analysis of $\mathrm{CO}_{2}$ mixtures by NDIR, mainly due to the matrices of the mixtures. During the analytical verification of the mixtures it was highlighted that NDIR analysers can have different responses depending on the 
matrix of the reference mixtures used for their calibration. Figure 2 shows the analytical reassignment of the molar fraction of a $\mathrm{CO}_{2}$ mixture in synthetic air prepared at INRIM having nominal value of $480.0 \mu \mathrm{mol}$ $\mathrm{mol}^{-1}$. It can be seen that the NDIR calibration with reference mixtures in synthetic air gives analytical values in agreement with the gravimetric value of the analysed mixture. On the other hand, a calibration with reference mixtures in a $\mathrm{N}_{2}$ matrix leads to an underestimation of the molar fraction value of the mixture.

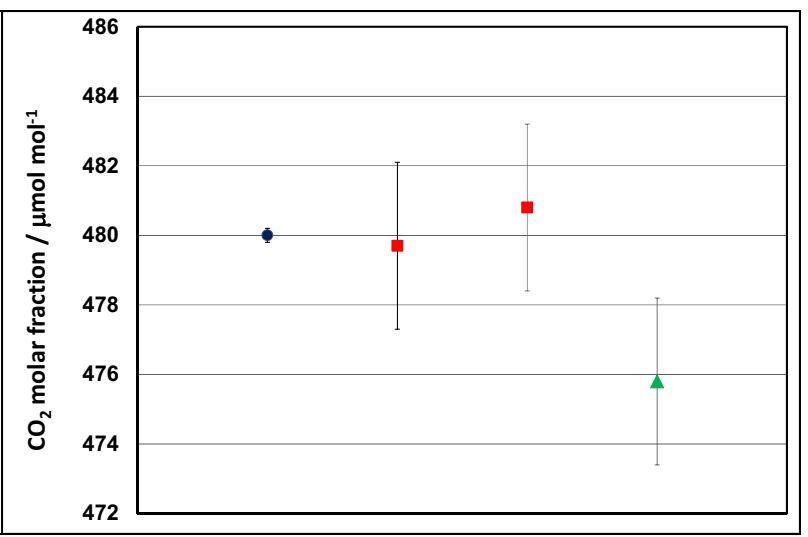

Fig. 2. Verification by NDIR analysis of the molar fraction of $\mathrm{CO}_{2}$ for a gravimetrically prepared mixture of $\mathrm{CO}_{2}$ at 480.0 $\mu \mathrm{mol} \mathrm{mol}^{-1}$ in synthetic air. $\bullet$ : gravimetric value; $\mathrm{n}$ : analytical value determined by NDIR calibrated with mixtures of $\mathrm{CO}_{2}$ in synthetic air; $\Delta$ : analytical value determined by NDIR calibrated with mixtures of $\mathrm{CO}_{2}$ in $\mathrm{N}_{2}$.

The opposite behaviour can be seen in figure 3: the molar fraction of a $\mathrm{CO}_{2}$ mixture in $\mathrm{N}_{2}$ purchased from a commercial supplier and having nominal value of 500.6

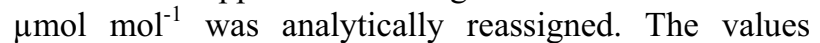
obtained after calibrating the NDIR with reference mixtures in $\mathrm{N}_{2}$ are in agreement with the certified value of the analysed mixture. A calibration with reference mixtures in synthetic air matrix leads instead to an overestimation of the molar fraction value of the mixture.

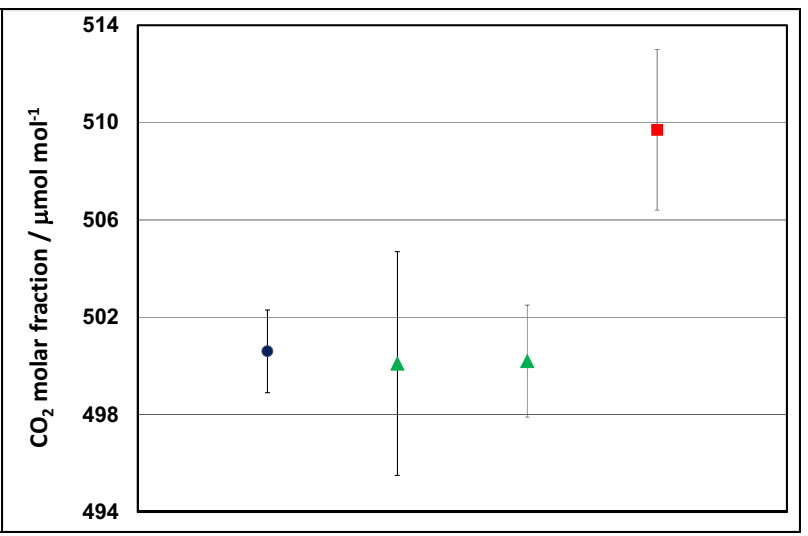

Fig. 3. Verification by NDIR analysis of the molar fraction of $\mathrm{CO}_{2}$ for a gravimetrically prepared mixture of $\mathrm{CO}_{2}$ at 500.6 $\mu \mathrm{mol} \mathrm{\textrm {mol } ^ { - 1 }}$ in $\mathrm{N}_{2}$. $\bullet$ : gravimetric value; $\boldsymbol{\Delta}$ : analytical value determined by NDIR calibrated with mixtures of $\mathrm{CO}_{2}$ in $\mathrm{N}_{2}$; $\mathbf{m}$ : analytical value determined by NDIR calibrated with mixtures of $\mathrm{CO}_{2}$ in synthetic air.
The data shown in Figures 2 and 3 prove that the matrix composition of the mixtures influences the analytical response of the NDIR photometers, hence they have to be calibrated by using suitable reference standards having a matrix as closest as possible to the mixture to be analysed or verified.

NDIR photometry is used to carry out stability studies on mixtures gravimetrically prepared at INRIM and also on standard mixtures commercially available. Figure 4 shows an example of the stability study on a mixture of $\mathrm{CO}_{2}$ at $360.0 \mu \mathrm{mol} \mathrm{mol}{ }^{-1}$ in synthetic air prepared at INRIM by gravimetry. It can be seen that the mixture shows long-term stability over a period of about 8 years.

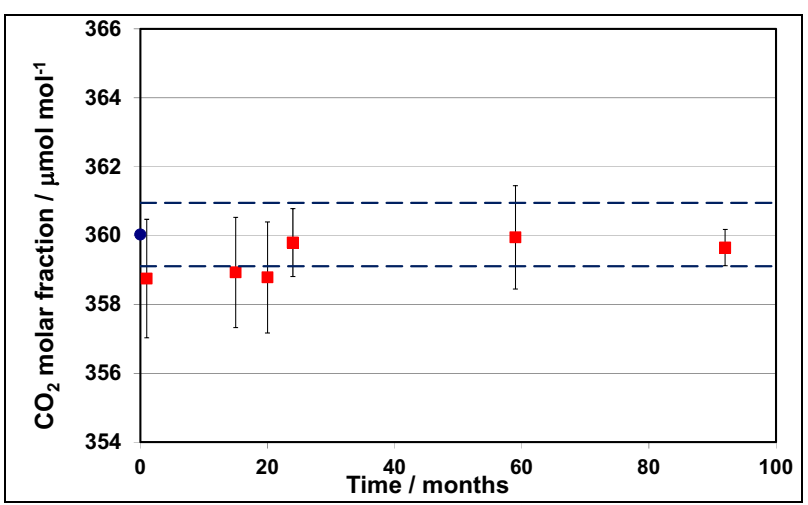

Fig. 4. Stability study of a mixture of $\mathrm{CO}_{2}$ at $360.0 \mu \mathrm{mol} \mathrm{mol}^{-1}$ in synthetic air gravimetrically prepared at INRIM.

๑: gravimetric value; $₫$ : analytical value determined by NDIR.

\section{Conclusions}

At INRIM, the dynamic dilution approach to generate $\mathrm{CO}_{2}$ reference gas mixtures at ambient level has recently been developed to support the well-established gravimetric preparation. NDIR is currently used to analytically verify and to carry out stability studies on gravimetric mixtures. The dynamic dilution can be applied to calibrate the NDIR photometers. A crucial point is the choice of suitable calibration mixtures both in terms of molar fraction and matrix composition. Research activities are also ongoing to improve different crucial points that might impact on the uncertainty evaluation, in particular on the verification and stability contributions: the identification of impurities represents a major issue. NDIR is selective to $\mathrm{CO}_{2}$ and can be easily used to determine the presence of $\mathrm{CO}_{2}$ impurities in matrix gases. Fourier Transform Infrared spectrometry can be applied for the identification of other impurities and degradation products both in the parent gases and in the prepared gas mixtures. Those compounds might affect the nominal concentration of the mixtures, their stability and also the response of the analysers.

\section{Acknowledgements}

The authors would like to thank Dr. Pier Giorgio Spazzini for his fundamental contribution in the development of the dynamic dilution system. 


\section{References}

1. World Meteorological Organization website http://www.wmo.int/pages/prog/arep/gaw/gaw hom e en.html (accessed on 30.06.2017)

2. V. Kitidis, I. Brown, N. Hardman-Mountford, N. Lefèvre, Prog. Oceanogr., (2016), http://dx.doi.org/10.1016/j.pocean.2016.08.005

3. E. Amico di Meane, M. Plassa, F. Rolle, M. Sega, Accred Qual Assur, 14, 607-611 (2009)

4. P. G. Spazzini, F. Pennecchi, E. Pessana, A. Piccato 9th International Symposium on Fluid Flow Measurement, Arlington (Virginia) (2015)

5. International Standard ISO 6142-1 "Gas analysis Preparation of calibration gas mixtures - Part 1 : Gravimetric method for Class I mixtures", Geneva ISO (2015)

6. F. Rolle, E. Pessana, M. Sega, J. Phys.: Conf. Ser. 841, 012032 (2017)

7. A. Malengo, F. Pennecchi, P. G. Spazzini, Calibration Curves Computing - Software for the evaluation of instrument calibration curves, Version 1.3, (2015), https://www.inrim.eu/researchdevelopment/quality-life/ccc-software (accessed on 30.06.2017) 